\title{
Recombinant human hyaluronidase facilitated subcutaneous immunoglobulin treatment in pediatric patients with primary immunodeficiencies: long-term efficacy, safety and tolerability
}

\begin{abstract}
Aim: To assess the long-term efficacy, safety and tolerability of recombinant human hyaluronidase-facilitated subcutaneous infusion of immunoglobulin (Ig) (fSCIG; HYQVIA $^{\oplus} ;$ IGHy) in children aged $<18$ years. Patients \& methods: Patients with primary immunodeficiency diseases were included in the studies. IGHy was administered every 3 or 4 weeks. Results: Validated acute serious bacterial infections were reported at $0.08 /$ patient-year (four pneumonia episodes in three patients). No serious adverse drug reaction (ADR) was reported, and rates of local and systemic ADRs were low (0.09/infusion and 0.1/infusion). Infection rates were low (3.02/patient-year) with sustained Ig trough levels (median: $1009 \mathrm{mg} / \mathrm{dl}$ ). Of 674 IGHy infusions, $97.2 \%$ required no change of administration due to ADR, in most ( $82.5 \%$ ) with one infusion site. No patient developed neutralizing anti-rHuPH20 antibodies. Postpivotal study, $100 \%$ of patients aged $<14$ years or their caregivers and $85.7 \%$ of patients aged 14 to $<18$ years expressed preference for IGHy compared with Ig administered intravenously or Ig administered subcutaneously. Conclusion: These studies, with the longest (maximum: 3.3 years) duration of any reported Ig replacement trials in children with primary immunodeficiency diseases, showed low infection, local and systemic reaction rates along with well-tolerated infusions given in a single site.
\end{abstract}

First draft submitted: 16 May 2016; Accepted for publication: 12 July 2016;

Published online: 28 July 2016

Keywords: home infusion $\bullet$ hyaluronidase $\bullet$ immunoglobulin $\bullet$ IVIG $\bullet$ PIDD • primary immunodeficiency disease

Children who have primary immunodeficiency diseases (PIDD) with antibody production defects [1] require treatment with immunoglobulin (Ig) administered intravenously (IV) or subcutaneously (SC). Ig replacement therapy has been used in primary antibody deficiencies for more than six decades [2,3]. Recently, patient centered treatment approaches have emphasized individually adjusted regimens based on patients' needs and activity levels. Both intravenous and subcutaneous Ig administration routes are effective and have inherent advantages and disadvantages: Ig given via the intravenous route (IVIG) involves few infusion sites and less frequent dosing (typically every 3-4 weeks); however, it requires venous access and the incidence of systemic adverse reactions is higher [4]; Ig administered subcutaneously (SCIG) can be self-administered at home, does not require venous access and is associated with fewer systemic reactions. It does, however, require more frequent dosing (typically weekly) and multiple sites and needle sticks per infusion. Recombinant human hyaluronidase ( $\mathrm{rHuPH} 20$ )-facilitated subcutaneous Ig infusion offers a novel treatment approach to control infections using fewer infusions and needle sticks per infusion than with conventional SCIG [5].

Immunoglobulin treatment, which is usually life-long in PIDD, constitutes a burden
Richard L Wasserman', Isaac Melamed², Lisa Kobrynski, Jennifer Puck ${ }^{4}$, Sudhir Gupta ${ }^{5}$, Jennifer Doralt ${ }^{6}$, Marlies Sharkhawy ${ }^{6}$, Werner Engl ${ }^{6}$, Heinz Leibl ${ }^{6}$, David Gelmont ${ }^{7}$ \& Leman Yel ${ }^{*, 7,5}$

${ }^{1}$ Allergy Partners of North Texas Research, Dallas, TX, USA 2IMMUNOe Health Centers, Centennial, CO, USA

${ }^{3}$ Emory University, Atlanta, GA, USA ${ }^{4}$ University of California, San Francisco, San Francisco, CA, USA

${ }^{5}$ University of California, Irvine, Irvine, CA, USA

${ }^{6}$ Baxalta Innovations $\mathrm{GmbH}$, now part of Shire, Vienna, Austria

${ }^{7}$ Baxalta US Inc., now part of Shire, Cambridge, MA, USA

*Author for correspondence: leman.yel@shire.com
Future $\because$.
Medicine $\%$ part of 
for patients. Venous access and its maintenance are challenging in children due to their small veins and inability to be cooperative for painful procedures [6]. Furthermore, the need for medical supervision may require travel to a site of care and potential systemic reactions may necessitate additional attention. Conventional SCIG can help address these issues, but the limited volume that can be infused into a single subcutaneous site usually requires the use of multiple infusion sites, commonly on a weekly basis $[7,8]$. The increased number of infusion sites and more frequent dosing may negatively impact quality of life and adherence to treatment [9]. Recombinant human hyaluronidase (rHuPH20)-facilitated subcutaneous infusion of Ig (fSCIG; IGHy; HYQVIA) has been developed to enable subcutaneous administration of volumes of Ig with a dose similar to intravenous administration $(300-600 \mathrm{mg} / \mathrm{kg})$ at a single site.

$\mathrm{rHuPH} 20$, a soluble form of naturally occurring human hyaluronidase, is suitable for chronic use in humans and is safe and effective at facilitating absorption and dispersion of subcutaneously administered fluids and drugs [10-13]. Preclinical studies have demonstrated that $\mathrm{rHuPH} 20$ is short-acting, with a halflife of less than $30 \mathrm{~min}$ when given subcutaneously. It is not detectable in plasma following administration at the doses utilized in subcutaneous infusions $[5,10]$. The safety and efficacy of rHuPH20 has been demonstrated in the augmentation of subcutaneous infusion of fluids and electrolytes to treat dehydrated pediatric patients $[13,14]$.

The use of rHuPH20 to facilitate SCIG was examined in a pivotal Phase III study [5] and its extension in adults and children treated every $3-4$ weeks [15]. In this manuscript, we focus on pediatric subjects from these studies presenting data from analyses that are distinct in scope.

\section{Patients \& methods \\ Study design}

The pivotal study and its extension study were consecutive, Phase III, prospective, open-label, noncontrolled, multicenter clinical studies in adults and children with PIDD [5,15-16]. Long-term safety, tolerability and efficacy of IGHy treatment in the pediatric subpopulation were examined using the pooled data from those studies. Subjects who completed the pivotal study were eligible to enroll in the extension study. These pivotal and extension studies were registered in ClinicalTrials.gov (NCT00814320 and NCT01175213). Informed consent forms and protocols were reviewed and approved by ethics committees, and informed consent was obtained from all patients and/or their legally authorized representatives before any study-related procedures began.
Assent was obtained from minor subjects according to local regulations. These analyses consider patients' age ( $<18$ years) based on their age at the time of subject screening for the pivotal study. The studies were performed in accordance with the International Conference on Harmonization Good Clinical Practice, the principles of the Declaration of Helsinki and applicable legal requirements and registered on ClinicalTrials.gov (NCT00814320 and NCT01175213).

\section{Study population}

Patients aged over 2 years with PIDD requiring antibody replacement as defined by the International Union of Immunological Societies [17,18] were eligible to participate in the pivotal study if they had been receiving Ig for $>3$ months before enrollment at a dose of $\geq 300 \mathrm{mg} / \mathrm{kg}$ of body weight $/ 4$ weeks. Completion of the pivotal study was a prerequisite for inclusion in the extension study.

\section{Treatment}

Normal human immunoglobulin $10 \%$ stabilized with glycine (GAMMAGARD LIQUID in the USA/Canada; elsewhere KIOVIG; Baxalta US Inc., CA, USA) was administered intravenously (referred to as IVIG) and subcutaneously in combination with $\mathrm{rHuPH} 20$ (IGHy) at a minimum dose ratio of $75 \mathrm{U} \mathrm{rHuPH} 20 / \mathrm{g}$ of IgG (Halozyme Therapeutics, Inc., CA, USA).

\section{Anti-rHuPH20 antibody assays}

Plasma samples were assessed for the presence of antirHuPH20 antibodies using a four-tiered testing strategy. In the first three tiers, plasma samples were tested for the presence of binding antibodies using a validated immunoreactivity assay employing a bridging format. This included an initial screen, a confirmatory test (i.e., competitive binding), and finally, determination of an end point antibody titer for only those samples deemed positive after the initial screen and confirmatory steps. The assay threshold is approximately $150 \mathrm{pg} / \mathrm{ml}$, which is more sensitive than most commonly used antidrug antibody assays [18]. Samples deemed positive were also tested for hyaluronidase neutralizing activity using a validated functional enzyme-inhibition assay.

\section{End points}

Efficacy was assessed during IGHy dosing in the pivotal and extension studies. The primary outcome measure was the rate of validated acute serious bacterial infections (VASBIs) per year. Additional efficacy end points included the rate of any infection, days off school/work, days on antibiotics, number of nonstudy outpatient visits, number of hospitalizations and days in hospital. 
Safety and tolerability were monitored throughout the duration of the two studies. End points assessed included the annual rate of adverse drug reactions (ADRs) defined as adverse events assessed by the investigator as related to treatment, rates of ADRs by patient and by infusion, categorization of ADRs by seriousness, severity, causality and temporal association with study treatment.

Study patients were monitored for the development of anti-rHuPH20 binding or neutralizing antibodies, and an association between antibody formation and clinical or laboratory ADRs was assessed.

In the extension study, the effect of varying IGHy infusion frequency on Ig trough levels was also assessed. Specific antibodies to relevant pathogens at the end of IVIG treatment in the pivotal study and at the end of IGHy treatment in the extension study were determined.

Treatment preference, an exploratory end point, was studied by surveying patients/caregivers at the completion of IGHy treatment.

\section{Statistical analysis}

Local, systemic and related adverse events and infections per patient per year were calculated for the entire study population and stratified for $<18$ and $\geq 18$ years old subjects in both studies.

At the end of IGHy infusion cycles, trough levels of Ig were analyzed in relation to dose frequency. Median trough levels were calculated based on infusion intervals.

\section{Results}

\section{Patient disposition}

A total of 26 patients aged $<18$ years enrolled at ten sites in the USA and Canada in the pivotal Phase III, prospective, open-label study lasting approximately 17 months for each patient [5]. Although patients from 2 years of age were eligible for the study, the youngest patient was 4 years old (Table 1). Two patients discontinued the study before starting on IGHy. Of the subjects who received IGHy $(n=24)$, five patients withdrew or were discontinued from the pivotal study. Of those five patients, two withdrew due to the adverse events: adenoidal hypertrophy $(\mathrm{n}=1)$ and mild infusion site pain $(n=1)$. The other three patients withdrew due to geographic factors $(n=2)$ and fear of needle sticks $(\mathrm{n}=1)$.

Nineteen pediatric patients completed 14-18 months of IGHy treatment in the pivotal study; of these, 15 patients opted to continue in the extension study. Of the 15 pediatric patients who began the extension study, three discontinued during IGHy treatment due to study site closure $(\mathrm{n}=2)$ and nonmedical personal reasons $(\mathrm{n}=1)$. The remaining 12 patients completed the extension study.

\section{Immunoglobulin treatment}

The total exposure for all pediatric patients in the combined studies was 48.7 patient-years. Patients who participated in both studies $(\mathrm{n}=15)$ received treatment for a median of 2.46 years (mean: 2.0 years; range: $0.1-3.3$ years).

During the pivotal and extension studies, patients received a median of 33.5 total infusions (mean: 30.8 infusions; min-max: 1-70 infusions), and the total number of IGHy infusions excluding the ramp-up period was 674. Of these, 655 (97.2\%) did not require any interruption, stopping or rate reduction of infusion due to ADRs. Most IGHy infusions including the ramp-up period were completed using only a single infusion site $(82.5 \% ; 609 / 738)$. Maximum volumes infused per site were, per protocol, dependent on body weight (Figure 1A \& B). Mean IGHy volume per site was $246 \mathrm{ml}$; volumes administered were lower in children aged 4 to $<12$ years (mean: $176 \mathrm{ml}$; range: $22-313 \mathrm{ml}$ ) than in adolescents aged 12 to $<18$ years (mean: $306 \mathrm{ml}$; range: $41-616 \mathrm{ml}$ ).

The mean IGHy doses were 0.655 and $0.570 \mathrm{~g} / \mathrm{kg} / \mathrm{month}$ among subjects aged 4 to $<12$ years and 12 to $<18$ years, respectively, and $0.616 \mathrm{~g} / \mathrm{kg} / \mathrm{month}$ among all pediatric subjects (Table 2). The average weight of subjects aged 4 to $<12$ years was $33.0 \mathrm{~kg}$; the average weight of those aged 12 to $<18$ years was $67.9 \mathrm{~kg}$. Based on body weight, doses were comparable among pediatric age groups: among those aged 4 to $<12$ years $(n=13)$, total mean dose per $\mathrm{kg}$ body weight was $0.455 \mathrm{~g} / \mathrm{kg}$ (SD: $0.1853)$, and among those aged 12 to $<18$ years $(\mathrm{n}=11)$, the dose was $0.470 \mathrm{~g} / \mathrm{kg}$ (SD: 0.1348 ).

The median maximum infusion rate was $300 \mathrm{ml} / \mathrm{h}$ overall, and $160 \mathrm{ml} / \mathrm{h}$ among younger subjects, aged 4 to $<12$ years. The median infusion time was $1.65 \mathrm{~h}$ (min-max: 1.17-2.68) for IGHy $(\mathrm{n}=24)$, compared with $2.42 \mathrm{~h}$ for IVIG $(\mathrm{n}=26)$.

\section{Table 1. Patient characteristics.}

\begin{tabular}{|ll|}
\hline Variable & Age $<18(\mathbf{n}=\mathbf{2 6})$ \\
Sex, $\mathrm{n}(\%)$ & $15(57.7)$ \\
- Male & $11(42.3)$ \\
- Female & $11.0(4-17)$ \\
\hline Age, years, median (range) & $42.0(15-117)$ \\
\hline Weight, median, kg (range) & \\
\hline $\begin{array}{l}\text { Two patients aged <18 years were not included in the analysis due to discontinuations } \\
\text { prior to receiving IGHy (patient request for withdrawal [ }=1] \text { and missed consecutive IVIG } \\
\text { administrations [ }=1]) \text {. In addition, two patients participated in only the initial ramp-up } \\
\text { period, during which frequency of IGHy therapy was gradually decreased, but then did not } \\
\text { continue in the study, due to mild infusion site pain }(n=1) \text { and geographical factors }(n=1) .\end{array}$ \\
\hline
\end{tabular}




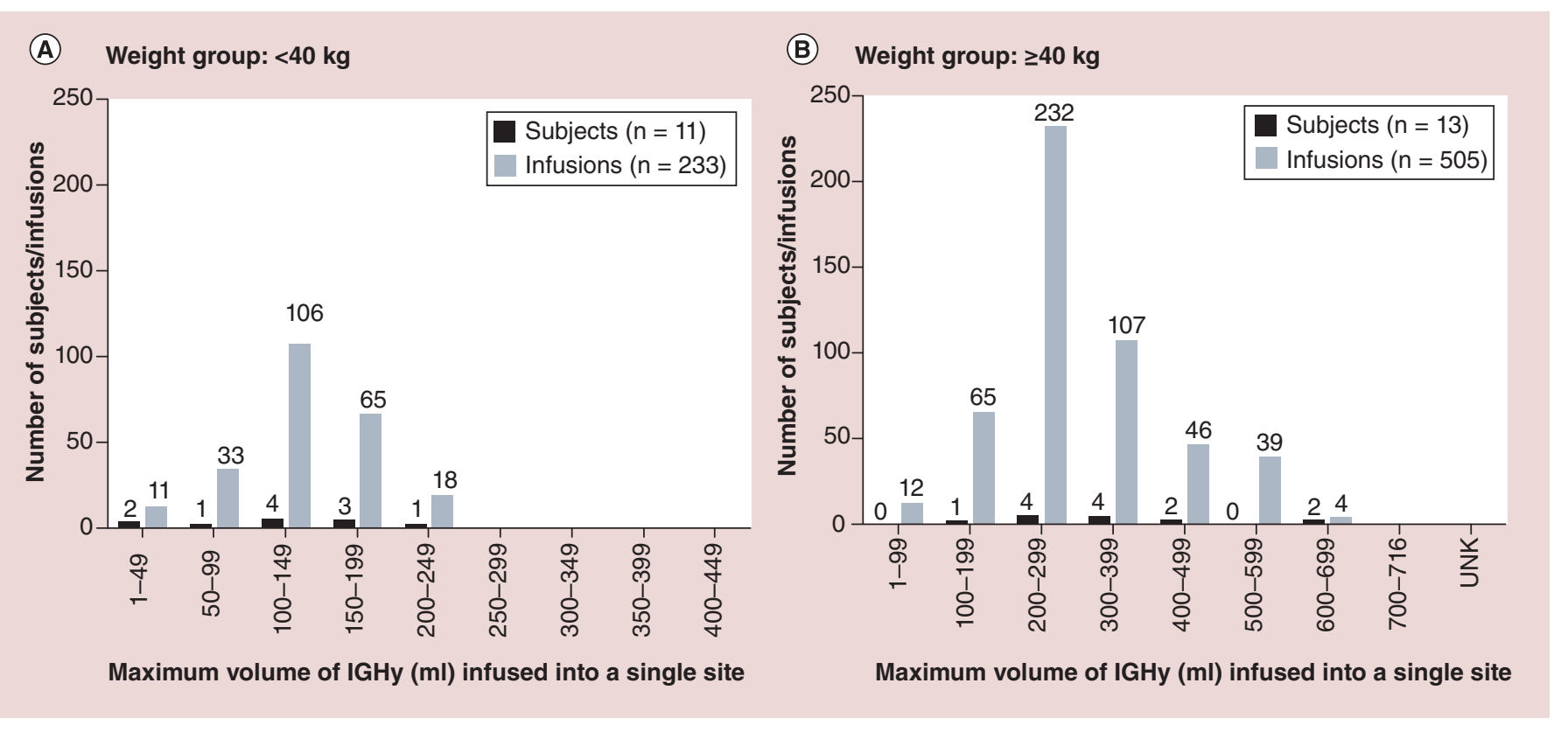

Figure 1. Summary of maximum IGHy volumes infused into a single site, among patients who weighed $<40 \mathrm{~kg}$ (A) and $>40 \mathrm{~kg}$ (B) at beginning of pivotal study. The protocol predefined the volume to be administered per site; for subjects weighing $<40 \mathrm{~kg}$, the maximum volume was $300 \mathrm{ml} / \mathrm{site}$ and for subjects weighing $>40 \mathrm{~kg}$, it was up to $600 \mathrm{ml} / \mathrm{site}$. Higher volumes were given in a few patients at the discretion of the investigator.

Most pediatric patients were infused with IGHy at 4-week intervals (18/24). Median steady state trough levels over the pivotal and extension studies combined were similar for the 3- and 4-week regimens $(1094 \mathrm{mg} / \mathrm{dl}$ [95\% CI: 914-1520 ( $=4)]$ and $1009 \mathrm{mg} / \mathrm{dl}$ [95\% CI: 852-1200], respectively) and were higher for the 2-week regimen relative to the 3or 4-week dosing intervals $(1660 \mathrm{mg} / \mathrm{dl}$ [95\% CI: NA due to low number of subjects $(\mathrm{n}=2)$ ]) (Table 3). In all patients, trough IgG levels after IGHy administration were comparable to the baseline levels prior to IGHy administration $(1020 \mathrm{mg} / \mathrm{dl}$ [min-max: 609-1780 $\mathrm{mg} / \mathrm{dl}]$ ), and well above the $500 \mathrm{mg} / \mathrm{dl}$ level which was considered the minimum target for IgG replacement at all treatment intervals $[19,20]$.

\section{Safety}

Six serious adverse events (headache, tonsillar enlargement, respiratory failure, status epilepticus, abdominal adhesion and pneumonia) were reported during the entirety of both studies. None was assessed as related to IGHy. A total of 67 local (0.09/infusion) and 70 systemic ADRs (0.10/infusion) considered related to infusions by the investigator were reported; all were mild or moderate in severity. Rates of local ADRs per infusion were lower among patients aged $4-<12(0.077)$ than among older patients (aged 12 to $<18$ years: 0.103; Table 3). Over the long observation period in the two studies (max 3.3 years), trends were examined in the rates of local and systemic reactions. The rate of local reactions appeared to decline over time, while the frequency of systemic reactions remained unchanged (data not shown).

All local reactions were mild $(n / n=47 / 67)$ or $\bmod$ erate (20/67) in severity. The most frequently reported local reactions were discomfort/pain and erythema with rates of 0.051 /infusion and 0.014/infusion, respectively. Infusion site swellings were transient and

Table 2. Immunoglobulin $10 \%$ dose per month per body weight administered subcutaneously with rHuPH20 for subjects $<18$ years in the pivotal and extension studies.

\begin{tabular}{|c|c|c|c|c|c|c|c|}
\hline \multirow[t]{2}{*}{ Age group } & \multicolumn{3}{|c|}{$\begin{array}{c}\text { Total dose } / \mathrm{kg} \text { body weight } / \text { month } \\
\text { (g/kg/month) }\end{array}$} & \multicolumn{3}{|c|}{ Total dose $/ \mathrm{kg}$ body weight $(\mathrm{g} / \mathrm{kg})$} & \multirow[t]{2}{*}{$\begin{array}{l}\text { Average weight } \\
\text { of subjects }(\mathrm{kg})\end{array}$} \\
\hline & $\begin{array}{l}\text { Number of } \\
\text { subjects }\end{array}$ & Mean & SD & $\begin{array}{l}\text { Number of } \\
\text { subjects }\end{array}$ & Mean & SD & \\
\hline Aged 4 to $<12$ years & 13 & 0.655 & 0.1929 & 13 & 0.455 & 0.1853 & 32.96 \\
\hline Aged 12 to $<18$ years & 11 & 0.570 & 0.1664 & 11 & 0.470 & 0.1348 & 67.88 \\
\hline Aged $<18$ years & 24 & 0.616 & 0.1826 & 24 & 0.462 & 0.1609 & 48.96 \\
\hline
\end{tabular}




\begin{tabular}{|c|c|c|c|c|c|c|c|c|}
\hline $\begin{array}{l}\text { Age group } \\
\text { (years) }\end{array}$ & Severity & $\begin{array}{l}\text { Total number } \\
\text { of ADRs }\end{array}$ & $\begin{array}{l}\text { Total number } \\
\text { of subjects }\end{array}$ & $\begin{array}{l}\text { Total number } \\
\text { of infusions }\end{array}$ & $\begin{array}{l}\text { Rate per } \\
\text { subject }\end{array}$ & $\begin{array}{l}\text { Rate per } \\
\text { infusion }\end{array}$ & $\begin{array}{l}\text { Subjects } \\
\text { experiencing } \\
\text { ADR, } n(\%)\end{array}$ & $\begin{array}{l}\text { Percentage of } \\
\text { infusions with } 1 \text { or } \\
\text { more ADRs, } \mathrm{n}(\%)\end{array}$ \\
\hline \multicolumn{9}{|l|}{ Local ADRs } \\
\hline \multirow[t]{4}{*}{4 to $<18$} & Mild & 47 & 24 & 738 & 1.958 & 0.064 & $13(54.2)$ & $41(5.6)$ \\
\hline & Moderate & 20 & 24 & 738 & 0.833 & 0.027 & $6(25.0)$ & $18(2.4)$ \\
\hline & Severe & 0 & 24 & 738 & 0.000 & 0.000 & $0(0.0)$ & $0(0.0)$ \\
\hline & Total & 67 & 24 & 738 & 2.792 & 0.091 & $13(54.2)$ & $55(7.5)$ \\
\hline 4 to $<12$ & Total & 26 & 13 & 339 & 2.000 & 0.077 & $7(53.8)$ & $22(6.5)$ \\
\hline 12 to $<18$ & Total & 41 & 11 & 399 & 3.727 & 0.103 & $6(54.5)$ & $33(8.3)$ \\
\hline \multicolumn{9}{|c|}{ Systemic ADRs } \\
\hline \multirow[t]{4}{*}{4 to $<18$} & Mild & 51 & 24 & 738 & 2.125 & 0.069 & $13(54.2)$ & $39(5.3)$ \\
\hline & Moderate & 19 & 24 & 738 & 0.792 & 0.026 & $6(25.0)$ & $12(1.6)$ \\
\hline & Severe & 0 & 24 & 738 & 0.000 & 0.000 & $0(0.0)$ & $0(0.0)$ \\
\hline & Total & 70 & 24 & 738 & 2.917 & 0.095 & $13(54.2)$ & $46(6.2)$ \\
\hline 4 to $<12$ & Total & 33 & 13 & 339 & 2.538 & 0.097 & $7(53.8)$ & $20(5.9)$ \\
\hline 12 to $<18$ & Total & 37 & 11 & 399 & 3.364 & 0.093 & $6(54.5)$ & $26(6.5)$ \\
\hline
\end{tabular}

resolved within $24-48 \mathrm{~h}$ after infusion. There were no clinically observable changes in the skin or subcutaneous tissue after 48.7 patient-years of total IGHy exposure.

The rate of related systemic reactions per infusion was 0.10 during IGHy treatment. The most frequently reported systemic ADRs during IGHy exposure were headache (0.030/infusion or $33.3 \%$ of patients), pyrexia (0.019/infusion or $20.8 \%$ of patients) and fatigue $(0.007 /$ infusion or $16.7 \%$ of patients; Table 4$)$.
Three patients (3/24: 12.5\%) aged 13, 14 and 17 years developed transient antibodies (titer $\geq 1: 160$ ) that bound to rHuPH20 (Figure 2). Their maximum titers were $1: 1280,1: 160$ and $1: 10240$ which decreased to $1: 40,1: 10$ and 1:320, respectively, at follow-up visits despite continued exposure to IGHy. These titers were similar to the baseline titers observed in $\mathrm{PH} 20$-naive healthy subjects $(\leq 1: 1280)$ [21]. These three patients did not have decreased IgG trough levels compared with their baseline levels, nor did they experience an

\begin{tabular}{|c|c|c|c|c|}
\hline \multirow[t]{2}{*}{ Preferred term } & \multicolumn{2}{|c|}{ IGHy } & \multicolumn{2}{|c|}{$\begin{array}{l}\text { Immune globulin infusion } 10 \% \text { (human) given } \\
\text { intravenously }\end{array}$} \\
\hline & $\begin{array}{l}\text { Number of subjects }(\%) \\
n=24\end{array}$ & $\begin{array}{l}\text { Number of ADRs per } \\
\text { infusion }\left(\text { rate }^{+}\right) n=738\end{array}$ & $\begin{array}{l}\text { Number of subjects }(\%) \\
n=26\end{array}$ & $\begin{array}{l}\text { Number of ADRs per infusion } \\
\left(\text { rate }^{+}\right) n=105\end{array}$ \\
\hline Headache & $8(33.3)$ & $22(0.030)$ & $11(42.3)$ & $26(0.248)$ \\
\hline Pyrexia & $5(20.8)$ & $14(0.019)$ & $3(11.5)$ & $4(0.038)$ \\
\hline Fatigue & 4 (16.7) & $5(0.007)$ & $3(11.5)$ & $3(0.029)$ \\
\hline Vomiting & $3(12.5)$ & $8(0.011)$ & $4(15.4)$ & $6(0.057)$ \\
\hline Pain & $2(8.3)$ & 7 (0.009) & $1(3.8)$ & $1(0.010)$ \\
\hline Nausea & $1(4.2)$ & $1(0.001)$ & $3(11.5)$ & $3(0.029)$ \\
\hline Chills & $0(0.0)$ & $0(0.000)$ & $3(11.5)$ & $5(0.048)$ \\
\hline $\begin{array}{l}\text { Heart rate } \\
\text { increased }\end{array}$ & $0(0.0)$ & $0(0.000)$ & $2(7.7)$ & $3(0.029)$ \\
\hline
\end{tabular}






Figure 2. Course of the anti-rHuPH20 antibodies in patients who developed positive titers $(n=3)$.

increase in the number of infections or any VASBI during the entire course of these studies. Annual rates of total, systemic and local ADRs after the detection of positive anti-rHuPH20 antibody titers $(\geq 1: 160)$ were lower or comparable to those prior to development of antibodies (Figure 3) [18]. An increased incidence in typical local adverse reactions such as induration, urticaria or evidence of local or systemic immune complex disease was not observed [21]. No patient developed neutralizing antibodies to $\mathrm{rHuPH} 20$.

\section{Efficacy (infections)}

During both studies that spanned up to 3.3 years, a total of four VASBIs were reported in three patients treated with IGHy; all VASBIs were pneumoniae. Two patients were treated for pneumonia with oral antibiotics. The third patient had Hyper-IgE syndrome and reported two episodes of staphylococcal pneumonia, each of which was treated as an in-patient. The IgG trough levels observed in these three patients were comparable to their baseline measurements (i.e., while receiving IVIG) and to those of other patients, with sufficient levels maintained throughout both studies. Overall, the VASBI rate (0.08/patient-year; upper limit of 99\% CI: 0.20) was significantly lower $(\mathrm{p}<0.0001)$ than the protocol-specified threshold of one VASBI/patient-year.

The rate of all infections occurring during IGHy exposure per patient-year $(\mathrm{n}=24)$ was $3.02(95 \% \mathrm{CI}$ : 2.15-4.10) and remained essentially the same throughout the combined studies, with point estimates of 3.39, 2.62 and 3.34 infections/year from months 1 to 12,13 to 24 and 25 to 33.6 , respectively, among all patients including the ones who withdrew from the studies. This trend was similar in all patients who completed both studies as well. The latter analysis was performed to avoid bias.

\section{Treatment preference}

After the pivotal study, all pediatric patients' caregivers, who responded when patients were aged $<14$ years, expressed a preference to continue IGHy treatment 
$(100.0 \%)$ rather than switch to IVIG or conventional SCIG (Figure 4). Of older adolescent patients ( $\geq 14$ years), most patients $(6 / 7 ; 85.7 \%)$ expressed a preference to remain on IGHy treatment, while one patient $(1 / 7 ; 14.3 \%)$ expressed a desire to return to regular subcutaneous treatment.

\section{Treatment location}

Although the majority of IGHy treatments were given at investigational sites in these studies, 45.5 and $20.0 \%$ of subjects, in the pivotal and extension studies, respectively, were treated with IGHy in the patients' own homes, in many cases without intervention by a healthcare professional.

\section{Discussion}

This was one of the longest clinical studies of Ig replacement therapy in pediatric patients published to date, with a total of 49 pediatric patient-years of IGHy treatment. No related serious ADRs and mostly mild local and systemic ADRs were reported in a substantial pediatric population, aged 4-17 years, despite subcutaneous administration of Ig at volumes and rates comparable to IVIG administration. These ADR rates were particularly encouraging considering that the infusion rate per site of $300 \mathrm{ml} / \mathrm{h}$ was 10 - to 15 -fold higher [5] and the volume per site was approximately 20 -fold higher than conventional SCIG administration [5,7,22-28].

Volumes of Ig comparable to IVIG administration, up to $300 \mathrm{ml} /$ site for those under $40 \mathrm{~kg}$, and up to $600 \mathrm{ml} / \mathrm{site}$ for those at or over $40 \mathrm{~kg}$, were administered (mean: $246 \mathrm{ml} / \mathrm{site}$; min-max: 22-616 ml/site). These volumes maintained Ig trough levels sufficient for protection against infections despite using administration intervals comparable to IVIG [29-31]: median steady state trough levels were $1094 \mathrm{mg} / \mathrm{dl}$ and $1009 \mathrm{mg} / \mathrm{dl}$ for the 3- and 4-week intervals, respectively, with a mean dose of $0.616 \mathrm{~g} / \mathrm{kg} /$ month, which compare favorably with patients' baseline (pre-IGHy treatment levels) and median trough levels observed after treatment with other subcutaneous and intravenous Ig preparations [19-20,22,24,32-37]. However, readers should exercise caution when comparing results between studies because of limitations due to different study designs, population demographics, treatment and dosing regimens.

A total of four VASBIs were reported in three pediatric patients treated with IGHy. The rate of VASBIs [38] per patient-year was low (0.08) compared with the regulatory agency required threshold of one VASBI/patient-year over the course of both the pivotal and extension studies, and also compared with those reported in older children and adults [27,29,32-33,39-40]. The rate of all infections per patient-year also remained

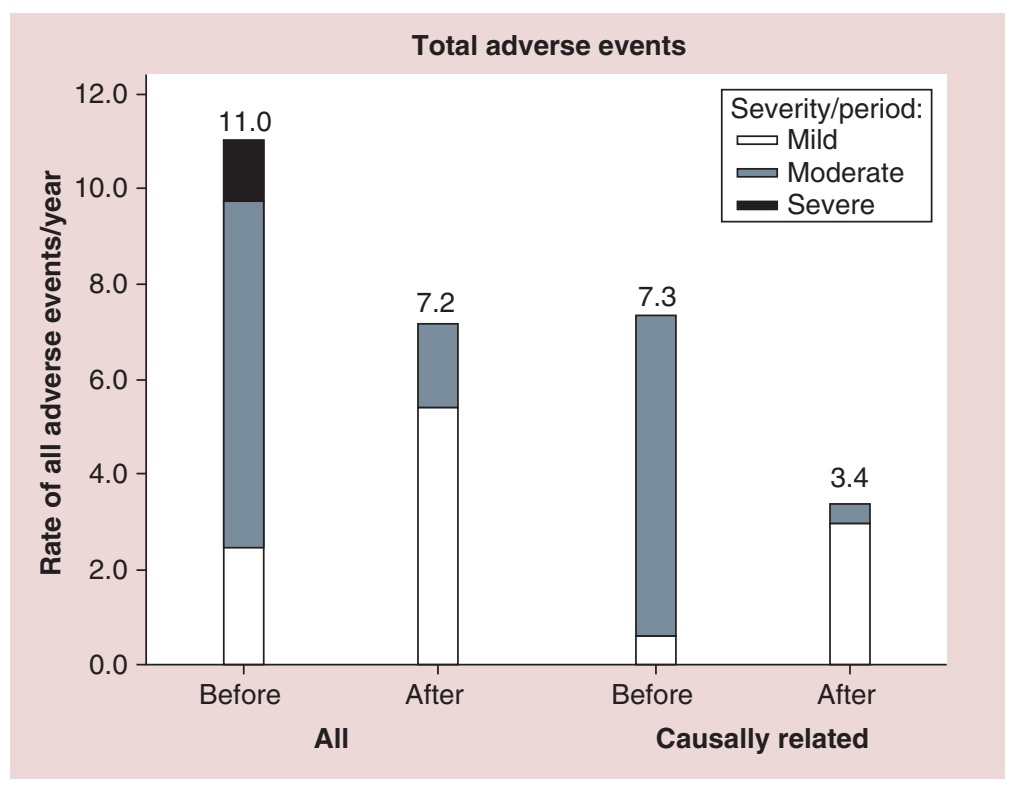

Figure 3. Summary of all related adverse events (excluding infections) by severity in subjects $(n=3)$ who developed anti-rHuPH20 antibodies. Rate $=$ number of adverse events divided by the years in the respective observation period. Before/after the date of the measurement which preceded the date of the first measurement with anti-rHuPH20 $\geq 160$.

low (3.07/pt/year) over the 3-year period studied. There was no indication that efficacy declined over time. Infection rates were consistently comparable to or lower than the previously reported rates with IVIG or SCIG administration in adults [7]. By comparison, pediatric patients aged $2-11$ receiving an SCIG 20\% product experienced 4.77 infections/year, and those aged 12-15 years experienced 5.18 infections/year [32].

A key element of this report, however, is the assessment of the safety of IGHy in pediatric patients, who

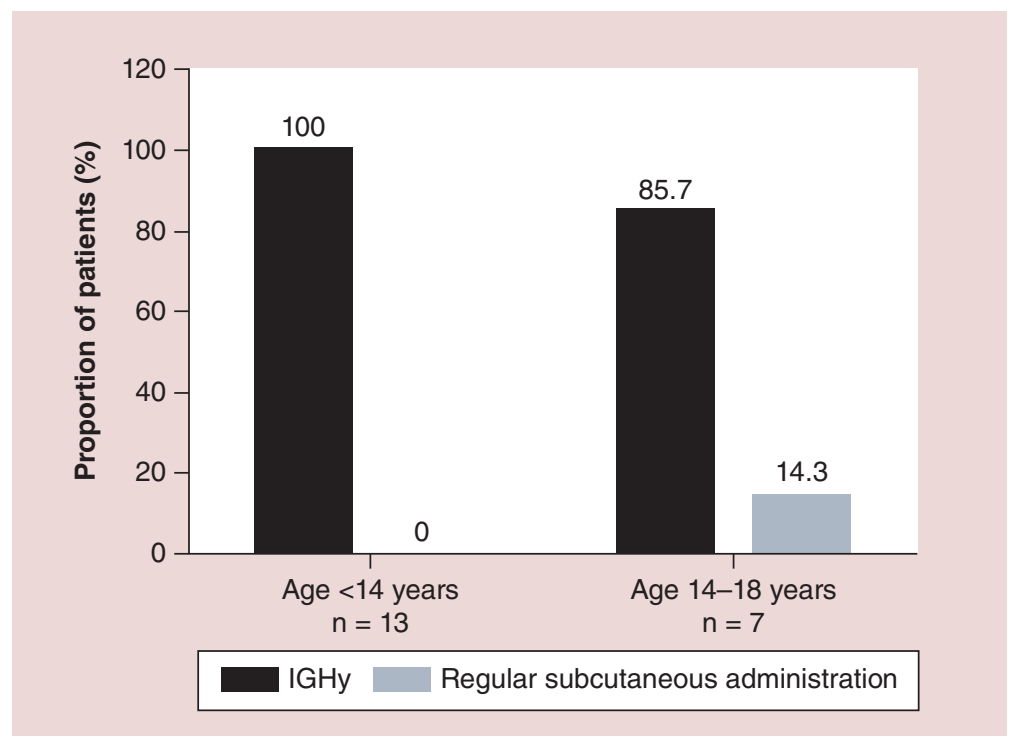

Figure 4. Subject or caregiver preference for Ig administered subcutaneous + rHuPH20, Ig administered subcutaneously or Ig administered intravenously at end of pivotal study. 
reported 0.091 local ADRs ( $\mathrm{n}=67$ over both studies) and 0.095 systemic ADRs $(\mathrm{n}=70)$ per infusion, and no related serious adverse events. The rate of local reactions appeared to decline over time, while the frequency of systemic reactions remained unchanged. This is consistent with previous reports of IGHy treatment in all age groups [7]. No severe, causally related local or systemic adverse events were reported; all were mild or moderate in severity. There were also no causally related serious adverse events reported. Infusion site swellings were temporary and resolved within $24-48 \mathrm{~h}$ after infusion. No clinically observable changes to the skin or subcutaneous tissue were reported. No patients developed neutralizing antibodies to $\mathrm{rHuPH} 20$ during either study. In fact, the patients with anti-rHuPH20 antibodies titers $\geq 1: 160$ showed isotype distribution and binding characteristics comparable to antibodies identified in healthy individuals without exposure to $\mathrm{rHuPH} 20[18,29]$. Furthermore, the titers of nonneutralizing antibodies declined over time, despite continued exposure to rHuPH20.

The prospect of safe and effective IGHy administration in children is particularly important because of the special needs of this population. Treatment of children was limited to IVIG or SCIG replacement therapy until recently, each of which has advantages and disadvantages of its own. The need for multiple needle sticks and weekly or biweekly administration of SCIG presents a special challenge for children with needle phobia. Conventional SCIG may require two to 20 injection sites per month with weekly administration [27], whereas most IGHy infusions (82.5\%) were completed using only a single infusion site, and $75 \%$ $(18 / 24)$ of the pediatric patients in this study were treated on a monthly basis. The advantages of a single infusion site at 3-4-week intervals would be even more significant in children [41].

In the present study, one pediatric patient withdrew from the pivotal study due to needle phobia. The reduced frequency of administration and the infusion at a single site with IGHy would be advantageous for sufferers of needle phobia that has the potential to significantly impact a child's quality of life and adherence. Needle phobia has been reported to affect $5-10 \%$ of the population [42,43], and $10 \%$ of pediatric patients (aged 6-17 years) with Type 1 diabetes mellitus patients, according to a recent study [44]. Conventional SCIG may require two to 20 infusion sites per month, compared with one in the majority of IGHytreated patients. This would represent an advantage in using IGHy in children with needle phobia.

IGHy administration was very well tolerated by pediatric subjects: $97.2 \%$ of 674 IGHy infusions were completed without reduction or interruption of administration. By comparison, children often require more than one attempt to establish venous access for administration of IVIG. In one of the largest studies of intravenous insertions in children, comprising 592 children and 1135 attempts at puncture carried out by 143 nurses, the failure rate was $10 \%$. The first attempted insertion was successful in fewer than half the children; in a third of attempts, the vein could not be cannulated even after two tries, and on average, each child was subjected to 2.1 attempts to obtain intravenous access. In addition, successful intravenous insertion took nearly $30 \mathrm{~min}$ [45] highlighting an advantage of subcutaneous administration.

The weekly dosing frequency of SCIG can be another treatment burden that is challenging for pediatric patients and caregivers and may lead to decreased adherence, particularly in adolescents. A review of 76 studies of patients across various therapeutic classes found that less frequent dosing regimens resulted in better adherence [46]. These observations were confirmed in treatment preferences after the pivotal study; $100.0 \%$ of patients' caregivers (if under 14 years old) and $85.7 \%$ of adolescent patients (aged 14 years or older) expressed a preference for continued treatment with IGHy compared with IVIG or conventional SCIG treatment at the end of the pivotal study.

\section{Conclusion}

IgG replacement therapy with IGHy, administered at 3-4 weekly intervals, was effective in maintaining Ig trough levels and preventing infections over a long period of time in pediatric patients with PIDD. It was very well tolerated; local and systemic reactions were almost all mild or moderate, and low in frequency. The majority of patients or their caregivers expressed a preference for IGHy treatment over alternative treatment options.

\section{Acknowledgements}

Figure $1 \mathrm{~A} \& \mathrm{~B}$ were prepared by $\mathrm{D}$ Lane; Figure 2 was prepared by E Jedrzejek (Baxalta US, Inc.). The authors are also grateful to N Nikolov, C Rabbat and T Berner for their critical review of the manuscript draft. The authors are grateful to all investigators who worked on these studies: R Roberts, J Routes, AJ Grant, R Schellenberg, J Church, M Stein, B Kettelhut, GW Richmond, K Kenneth, A Ratnayake and A Rubenstein.

\section{Financial \& competing interests disclosure}

W Engl, J Doralt, M Sharkhawy, H Leibl, D Gelmont and L Yel are full-time employees and shareholders of Baxalta, now part of Shire. RL Wasserman, I Melamed, L Kobrynski, J Puck and S Gupta worked as investigators on these studies, of which Baxalta, now part of Shire, was the sponsor. The authors have no other relevant affiliations or financial involvement with any 
organization or entity with a financial interest in or financial conflict with the subject matter or materials discussed in the manuscript apart from those disclosed.

No writing assistance was utilized in the production of this manuscript.

\section{Ethical conduct of research}

The authors state that they have obtained appropriate institutional review board approval or have followed the principles outlined in the Declaration of Helsinki for all human or animal experimental investigations. In addition, for investigations involving human subjects, informed consent has been obtained from the participants involved.

\section{Open access}

This work is licensed under the Attribution-NonCommercialNoDerivatives 4.0 Unported License. To view a copy of this license, visit http://creativecommons.org/licenses/by-nc-nd/4.0/

\section{Executive summary}

- IgG replacement therapy with recombinant human hyaluronidase-facilitated subcutaneous infusion of immunoglobulin (fSCIG; HYQVIA ${ }^{\oplus}$; IGHy), administered at 3-4 weekly intervals, was effective and safe over a long period of time (up to 3.3 years) in pediatric patients (aged $<18$ years) with primary immunodeficiency diseases.

- Validated acute serious bacterial infections were reported at a rate of $0.08 /$ patient-year, substantially lower than the specified threshold of $1 /$ patient-year $(p<0.0001)$.

- Infection rates were low (3.02/patient-year) and patients maintained stable IgG trough levels (median: $1009 \mathrm{mg} / \mathrm{dl})$.

- Of a total of 674 IGHy infusions, 655 (97.2\%) required no change of administration.

- Most IGHy infusions (82.5\%) were completed using only a single infusion site.

- $100.0 \%$ of patients' caregivers (if under 14 years old) and $85.7 \%$ of adolescent patients (aged 14 years or older) expressed a preference for continued treatment with IGHy compared with intravenously administered immunoglobulin or conventional subcutaneously administered immunoglobulin treatment at the end of the pivotal study.

\section{References}

Papers of special note have been highlighted as:

- of interest; $\bullet \bullet$ of considerable interest

1 Picard C, Al-Herz W, Bousfiha A et al. Primary immunodeficiency diseases. An update on the classification from the International Union of Immunological Societies Expert Committee for Primary Immunodeficiency 2015. J. Clin. Immunol. 35(8), 696-726 (2015).

2 Sriaroon P, Ballow M. Immunoglobulin replacement therapy for primary immunodeficiency. Immunol. Allergy Clin. North Am. 35(4), 713-730 (2015).

3 Wasserman RL. Progress in gammaglobulin therapy for immunodeficiency: from subcutaneous to intravenous infusions and back again. J. Clin. Immunol. 32(6), 1153-1164 (2012).

- Provides an overview of the options available for infusing immunoglobulin, with their advantages and disadvantages; the manuscript cites relevant studies and literature.

4 Abolhassani H, Sadaghiani MS, Aghamohammadi A, Ochs HD, Rezaei N. Home-based subcutaneous immunoglobulin versus hospital-based intravenous immunoglobulin in treatment of primary antibody deficiencies: systematic review and meta analysis. J. Clin. Immunol. 32(6), 1180-1192 (2012).

- Compares efficiency and safety of immunoglobulins infused subcutaneously or intravenously, and concludes that changing to subcutaneous immunoglobulin replacement therapy may be beneficial to qualified patients with primary immunodeficiency diseases.
5 Wasserman RL, Melamed I, Stein MR et al. Recombinant human hyaluronidase-facilitated subcutaneous infusion of human immunoglobulins for primary immunodeficiency. J. Allergy Clin. Immunol. 130(4), 951-957.e11 (2012).

-. Details the pivotal study referred to in this manuscript, in which $19 / 24$ pediatric patients completed treatment with IGHy.

6 Garcia-Lloret M, McGhee S, Chatila TA. Immunoglobulin replacement therapy in children. Immunol. Allergy Clin. North Am. 28(4), 833-849 (2008).

- Covers the common advantages and disadvantages of various methods of immunoglobulin replacement in pediatric patients. It was written before IGHy was developed, but the needs of the pediatric population have not changed dramatically since its writing.

7 Ochs HD, Gupta S, Kiessling P, Nicolay U, Berger M, Subcutaneous IgG Study Group. Safety and efficacy of self-administered subcutaneous immunoglobulin in patients with primary immunodeficiency diseases. J. Clin. Immunol. 26(3), 265-273 (2006).

8 Misbah S, Sturzenegger MH, Borte M et al. Subcutaneous immunoglobulin. Opportunities and outlook. Clin. Exp. Immunol. 158(Suppl. 1), 51-59 (2009).

9 Ponsford M, Carne E, Kingdon C et al. Facilitated subcutaneous immunoglobulin (fSCIg) therapy - practical considerations. Clin. Exp. Immunol. 182(3), 302-313 (2015).

- Describes the first 14 patients treated with IGHy at a center in Wales. It also provides practical guidance on 
initial experience with the administration, training and outcomes of IGHy therapy.

10 Frost GI. Recombinant human hyaluronidase (rHuPH20): an enabling platform for subcutaneous drug and fluid administration. Expert Opin. Drug Deliv. 4(4), 427-440 (2007).

- The volume of drug that can be injected at a single site, the rate and amount that reach the vascular compartment is limited by the extracellular matrix. Frost describes the application of the recombinant DNA-derived hyaluronidase enzyme ( $\mathrm{rHuPH} 20)$ technology that helps to increase injection volumes and bioavailability from subcutaneous injections.

11 Thomas JR, Yocum RC, Haller MF, von Gunten CF. Assessing the role of human recombinant hyaluronidase in gravity-driven subcutaneous hydration: The INFUSE-LR Study. J. Palliat. Med. 10(6), 1312-1320 (2007).

12 Harb G, Lebel F, Battikha J, Thackara JW. Safety and pharmacokinetics of subcutaneous ceftriaxone administered with or without recombinant human hyaluronidase (rHuPH20) versus intravenous ceftriaxone administration in adult volunteers. Curr. Med. Res. Opin. 26(2), 279-288 (2010).

13 Allen CH, Etzwiler LS, Miller MK et al. Recombinant human hyaluronidase-enabled subcutaneous pediatric rehydration. Pediatrics 124(5), e858-e867 (2009).

14 Spandorfer PR, Mace SE, Okada PJ et al. A randomized clinical trial of recombinant human hyaluronidasefacilitated subcutaneous versus intravenous rehydration in mild to moderately dehydrated children in the emergency department. Clin. Ther. 34(11), 2232-2245 (2012).

15 Wasserman RL, Melamed I, Stein MR et al. Long-term tolerability, safety and efficacy of recombinant human hyaluronidase-facilitated subcutaneous infusion of human immunoglobulin for primary immunodeficiency. J. Clin. Immunol. 36(6), 571-582 (2016).

- $\quad$ This is the primary publication detailing both the pivotal and extension studies referred to in this manuscript, which included both adult $(n=59)$ and pediatric $(n=24)$ patients.

16 Wasserman RL. Subcutaneous immunoglobulin: facilitated infusion and advances in administration. Clin. Exp. Immunol. 178(Suppl. 1), 75-77 (2014).

17 Geha RS, Notarangelo LD, Casanova JL et al. Primary immunodeficiency diseases. an update from the International Union of Immunological Societies Primary Immunodeficiency Diseases Classification Committee. J. Allergy Clin. Immunol. 120(4), 776-794 (2007).

18 Rosengren S, Dychter SS, Printz MA et al. Clinical immunogenicity of $\mathrm{rHuPH} 20$, a hyaluronidase enabling subcutaneous drug administration. AAPS J. 17(5), 1144-1156 (2015).

19 Orange JS, Grossman WJ, Navickis RJ, Wilkes MM. Impact of trough $\mathrm{IgG}$ on pneumonia incidence in primary immunodeficiency: a meta-analysis of clinical studies. Clin. Immunol. 137(1), 21-30 (2010).

20 Quinti I, Soresina A, Guerra A et al. Effectiveness of immunoglobulin replacement therapy on clinical outcome in patients with primary antibody deficiencies. results from a multicenter prospective cohort study. J. Clin. Immunol. 31(3), 315-322 (2011).

21 Baxter Bioscience. Briefing Book, Blood Products Advisory Committee Meeting, July 31, 2014, HyQvia Immune Globulin Infusion 10\% (Human) with Recombinant Human Hyaluronidase. 204 (2014). www.fda.gov

22 Wasserman RL, Melamed I, Kobrynski L et al. Efficacy, safety, and pharmacokinetics of a $10 \%$ liquid immune globulin preparation (GAMMAGARD LIQUID, 10\%) administered subcutaneously in subjects with primary immunodeficiency disease. J. Clin. Immunol. 31(3), 323-331 (2011).

23 Gardulf A, Björvell H, Gustafson R, Hammarström L, Smith CI. Safety of rapid subcutaneous gammaglobulin infusions in patients with primary antibody deficiency. Immunodeficiency 4(1-4), 81-84 (1993).

24 Hagan JB, Fasano MB, Spector S et al. Efficacy and safety of a new $20 \%$ immunoglobulin preparation for subcutaneous administration, IgPro20, in patients with primary immunodeficiency. J. Clin. Immunol. 30(5), 734-745 (2010).

25 Frost GI, Stern R. A microtiter-based assay for hyaluronidase activity not requiring specialized reagents. Anal. Biochem. 251(2), 263-269 (1997).

26 Baxalta Incorporated. Annex I Summary of product characteristics HyQvia $100 \mathrm{mg} / \mathrm{ml}$ solution for infusion for subcutaneous use. 43 (2015). www.ema.europa.eu

27 Patel NC, Gallagher JL, Ochs HD et al. Subcutaneous immunoglobulin replacement therapy with Hizentra ${ }^{\circledR}$ is safe and effective in children less than 5 years of age. J. Clin. Immunol. 35(6), 558-565 (2015).

28 Bayer Corporation. Package Insert, Immune Globulin Intravenous (Human) Gamunex. 16- (2003). www.baxter.ca

29 Wasserman RL, Melamed I et al., Nelson RP Jr Pharmacokinetics of subcutaneous IgPro20 in patients with primary immunodeficiency. Clin. Pharmacokinet. 50(6), 405-414 (2011).

30 Bonagura VR, Marchlewski R, Cox A, Rosenthal DW. Biologic IgG level in primary immunodeficiency disease: the IgG level that protects against recurrent infection. J. Allergy Clin. Immunol. 122(1), 210-212 (2008).

31 Stiehm ER. Human intravenous immunoglobulin in primary and secondary antibody deficiencies. Pediatr. Infect. Dis. J. 16(6), 696-707 (1997)

32 Borte M, Pac M, Serban M et al. Efficacy and safety of Hizentra $^{\circledR}$, a new $20 \%$ immunoglobulin preparation for subcutaneous administration, in pediatric patients with primary immunodeficiency. J. Clin. Immunol. 31(5), 752-761 (2011).

33 Church J, Borte M, Taki H et al. Efficacy and safety of Privigen in children and adolescents with primary immunodeficiency. Pediatr. Asthma Allergy Immunol. 22(2), 53-61 (2009).

34 Gardulf A, Nicolay U, Asensio O et al. Rapid subcutaneous $\mathrm{IgG}$ replacement therapy is effective and safe in children 
and adults with primary immunodeficiencies - a prospective multi-national study. J. Clin. Immunol. 26(2), 177-185 (2006).

\section{Wasserman RL, Church JA, Peter HH et al.}

Pharmacokinetics of a new $10 \%$ intravenous immunoglobulin in patients receiving replacement therapy for primary immunodeficiency. Eur. J. Pharm. Sci. 37(3-4), 272-278 (2009).

Wasserman RL, Irani AM, Tracy J et al. Pharmacokinetics and safety of subcutaneous immune globulin (human), 10\% caprylate/chromatography purified in patients with primary immunodeficiency disease. Clin. Exp. Immunol. 161(3), 518-526 (2010).

Stein MR, Nelson RP, Church JA et al. Safety and efficacy of Privigen ${ }^{\circledR}$, a novel $10 \%$ liquid immunoglobulin preparation for intravenous use, in patients with primary immunodeficiencies. J. Clin. Immunol. 29(1), 137-144 (2009).

38 U. S. Department of Health and Human Services, Food and Drug Administration, and Center for Biologics Evaluation and Research. Guidance for industry. Safety, efficacy, and pharmacokinetic studies to support marketing of immune globulin intravenous (human) as replacement therapy for primary humoral immunodeficiency. 17- (2008). www.fda.gov

Jolles S, Bernatowska E, De Gracia J et al. Efficacy and safety of Hizentra ${ }^{\circledR}$ in patients with primary immunodeficiency after a dose-equivalent switch from intravenous or subcutaneous replacement therapy. Clin. Immunol. 141(1), 90-102 (2011).

Wasserman RL, Church JA, Stein M et al. Safety, efficacy and pharmacokinetics of a new $10 \%$ liquid intravenous immunoglobulin (IVIG) in patients with primary immunodeficiency. J. Clin. Immunol. 32(4), 6637-6669 (2012).

41 Gardulf A, Nicolay U, Asensio O et al. Children and adults with primary antibody deficiencies gain quality of life by subcutaneous IgG self-infusions at home. J. Allergy Clin. Immunol. 114(4), 936-942 (2004).

-• This study, based on questionnaires submitted by pediatric and adult patients receiving intravenous (hospital-based) or subcutaneous treatment (at home) of immunoglobulin, demonstrates that home-based subcutaneous immunoglobulin therapy improves several aspects of health-related quality of life and provides patients with greater independence and better control of the therapy and daily life. For adults and children who are in need of lifelong immunoglobulin replacement therapy, subcutaneous immunoglobulin home therapy was found to be an appreciated therapeutic alternative.

42 Craske MG. Fear and anxiety in children and adolescents. Bull. Menninger Clin. 61(2 Suppl. A), A4-A36 (1997).

43 Kleinknecht RA. Acquisition of blood, injury, and needle fears and phobias. Behav. Res. Ther. 32(8), 817-823 (1994).

44 Cemeroglu AP, Can A, Davis AT et al. Fear of needles in children with type 1 diabetes mellitus on multiple daily injections and continuous subcutaneous insulin infusion. Endocr. Pract. 21(1), 46-53 (2015).

45 Larsen P, Eldridge D, Brinkley J et al. Pediatric peripheral intravenous access. Does nursing experience and competence really make a difference? J. Infus. Nurs. 33(4), 226-235 (2010).

46 Claxton AJ, Cramer J, Pierce C. A systematic review of the associations between dose regimens and medication compliance. Clin. Ther. 23(8), 1296-1310 (2001). 
